\title{
Unsteady Crack Motion and Branching in a Phase-Field Model of Brittle Fracture
}

\author{
Alain Karma and Alexander E. Lobkovsky \\ Department of Physics and Center for Interdisciplinary Research on Complex Systems, \\ Northeastern University, Boston, MA 02115
}

\begin{abstract}
Crack propagation is studied numerically using a continuum phase-field approach to mode III brittle fracture. The results shed light on the physics that controls the speed of accelerating cracks and the characteristic branching instability at a fraction of the wave speed.
\end{abstract}


The quest for a fundamental understanding of dynamic brittle fracture has been an ongoing challenge. The traditional continuum approach to computing the speed of brittle cracks consists of solving the equations of linear elasticity with boundary conditions on the moving fracture surfaces up to the crack tip 11]. The solutions have stress fields that diverge near the tip representing a finite energy flow rate to the tip. The crack speed $v$ is then assumed to be uniquely determined by this energy flow rate. In this theory, all the nonlinear physics of failure inside a microscopic region around the tip - the so-called process zone - is buried in a phenomenological function that relates the fracture energy $\Gamma$ of the material (energy needed to advance the tip per unit length of crack front and per unit of crack extension) and $v$.

Detailed experiments have been carried out to test this theory by measuring the speed of accelerating cracks with different initial lengths and different loads [2]. The theory should be valid if a unique $\Gamma(v)$ curve applies to the different cracks for the same material. The main difficulty in any experimental test of this theory is that the energy flux to the tip cannot be measured directly. Therefore, this flux needs to be inferred from a time-dependent solution of linear elasticity for accelerating cracks. Using an approximate solution, Sharon and Fineberg have concluded that the data for different cracks collapse on a unique $\Gamma(v)$ curve, whereas, using an exact solution of Eshelby [3], Kessler and Levine have concluded that this collapse does not occur [4].

Also of long-standing interest in brittle fracture is the existence of a dynamic instability that limits the speed of fast moving cracks. This generic instability has been seen in both experiments [2, 5, 6] and molecular dynamic simulations [7]. The fact that different amorphous materials with entirely different microscopic details (such as glass and PMMA) exhibit strikingly similar branching instabilities [2] strongly suggests that a continuum theory may be appropriate for understanding this phenomenon. Devising such a theory, however, has proven to be difficult. Cohesive zone theories modify the boundary conditions on the stress field near the tip to take into account the short scale force between crack surfaces. Langer and Lobkovsky 8], however, have shown that these models are unsuitable for stability calculations since the results depend singularly on the details of the cohesive zone. In addition, intrinsically discrete branching instabilities in lattice models [9, 10] seem unlikely to bear relevance to experiments in amorphous materials.

In this letter, we study dynamic brittle fracture using a recently developed continuum 
phase-field approach for mode III cracks [11]. This approach has the chief advantage that it incorporates both the short-scale physics of failure and macroscopic linear elasticity within a self-consistent set of coupled nonlinear partial differential equations that can be solved numerically. Moreover, solutions free of discretization artifacts can be obtained. Earlier simulations of this model were limited to small system sizes owing to the extreme stiffness of the equations, and no branching instability was found even for cracks approaching the wave speed [1]. Here, we briefly discuss how to overcome this stiffness and carry out simulations in systems large enough to make contact with results from the fracture community. In addition, these simulations enable us to study the onset of the characteristic branching instability at a fraction of the wave speed that turns out to be a robust feature of this phase-field model.

The basic variables of the model [1] are the scalar displacement $u(x, y)$ perpendicular to the $x-y$ plane of mass points from their original positions, and the phase-field, $\phi(x, y)$, which describes the state of the material. The unbroken solid, which behaves purely elastically, corresponds to $\phi=1$, whereas the fully broken material that cannot support stress corresponds to $\phi=0$. The total energy (kinetic plus elastic) of the system per unit length of the crack front is

$$
E=\int d x d y\left[\frac{\rho}{2}\left(\frac{\partial u}{\partial t}\right)^{2}+\frac{\kappa}{2}|\vec{\nabla} \phi|^{2}+h f(\phi)+\frac{\mu}{2} g(\phi)\left(|\vec{\epsilon}|^{2}-\epsilon_{c}^{2}\right)\right]
$$

where $\rho$ is the density, $\vec{\epsilon} \equiv \vec{\nabla} u$ is the strain, $f(\phi)$ is a double-well potential with minima at $\phi=1$ and $\phi=0, \mu$ is the elastic shear modulus, and $\epsilon_{c}$ is the critical strain magnitude such that the unbroken (broken) state is energetically favored for $|\vec{\epsilon}|<\epsilon_{c}\left(|\vec{\epsilon}|>\epsilon_{c}\right)$. The function $g(\phi)$ is a monotonously increasing function of $\phi$ with limits $g(0)=0$ and $g(0)=1$, which controls the softening of the elastic energy as more bonds are broken.

Taking the first variations of the energy with respect to the strain and to $\phi$, we obtain the stress, $\vec{\sigma}=\delta E / \delta \vec{\epsilon}$, and the equations of motion

$$
\begin{aligned}
\frac{\partial \phi}{\partial t} & =-\chi \frac{\delta E}{\delta \phi}, \\
\rho \frac{\partial^{2} u}{\partial t^{2}} & =\vec{\nabla} \cdot \vec{\sigma} .
\end{aligned}
$$

Energy is dissipated in the process zone around the crack tip where $\phi$ varies rapidly in space and time. Eq. (2a) implies that the size of the process zone is $\sim \xi=\sqrt{\kappa / \mu \epsilon_{c}^{2}}$ and 
the characteristic time of energy dissipation in this zone is $\tau=1 /\left(\chi \mu \epsilon_{c}^{2}\right)$. Furthermore, by rescaling lengths by $\xi$, time by $\xi / c$, where $c \equiv \sqrt{\mu / \rho}$ is the shear wave speed, and $u$ by $\xi \epsilon_{c}$ in Eqs. (2), we find that crack propagation is controlled by two dimensionless parameters $\delta=h /\left(\mu \epsilon_{c}^{2}\right)$ and $\beta=c \tau / \xi$. The first determines the surface energy normalized by $\mu \epsilon_{c}^{2} \xi$ [1]

$$
\widetilde{\gamma} \equiv \frac{\gamma}{\mu \epsilon_{c}^{2} \xi}=\int_{0}^{1} d \phi \sqrt{1-g(\phi)+2 \delta f(\phi)} .
$$

For the choices $f(\phi)=16 \phi^{2}(1-\phi)^{2}$ and $g(\phi)=4 \phi^{3}-3 \phi^{4}$ here, $\widetilde{\gamma}$ approximately doubles when $\delta$ increases from 0 to 2 .

Parameter $\beta$ controls the importance of inertia relative to dissipation in the process zone. When $\beta \ll 1$ (inertia-dominated regime), failure is rapid and crack propagation is governed by elastic energy flow. When $\beta \gg 1$ (dissipation-dominated regime), failure is sluggish. The elastic displacements are quasistatic and propagation is governed by the kinetics of the failure process.

We study fracture numerically in a strip of width $2 W$ with a fixed displacement $u(x, \pm W)= \pm \Delta$ at the strip edges. The stored elastic energy per unit area ahead of the crack tip is $G=\mu \Delta^{2} / W$ and the Griffith's threshold load for a semi-infinite crack is $G_{c}=2 \gamma$.

The equations of the model are inherently stiff due to strain localization behind the crack tip [11]. The strain is localized within a narrow region whose width vanishes in the large strip limit $W / \xi \rightarrow \infty$, while the width of the $\phi$ variation remains of order $\xi$ in this limit. Therefore, while it is possible to resolve the spatial variation of $\phi$ everywhere, resolving the strain concentration behind the crack tip is computationally impractical for large strip widths. The key to overcoming this difficulty is to recognize that the model remains welldefined when the strain variation is resolved only in the process zone, but is allowed to be discontinuous on the lattice scale far behind the tip. Therefore, it is possible to simply discretize the energy $E$ on a square lattice and to formulate the dynamics of the model from partial derivatives of $E$ with respect to the variables $\phi$ and $u$ at lattice points. One subtlety of the present simulations is that high-frequency waves are radiated behind the crack tip above a small threshold tip speed. These waves increase slightly the fracture toughness and originate from the region behind the tip where the displacement becomes discontinuous on the lattice scale. In addition, multiple reflections of these waves can cause tip oscillations at long times. This time, however, is longer than the time for propagation to reach a 


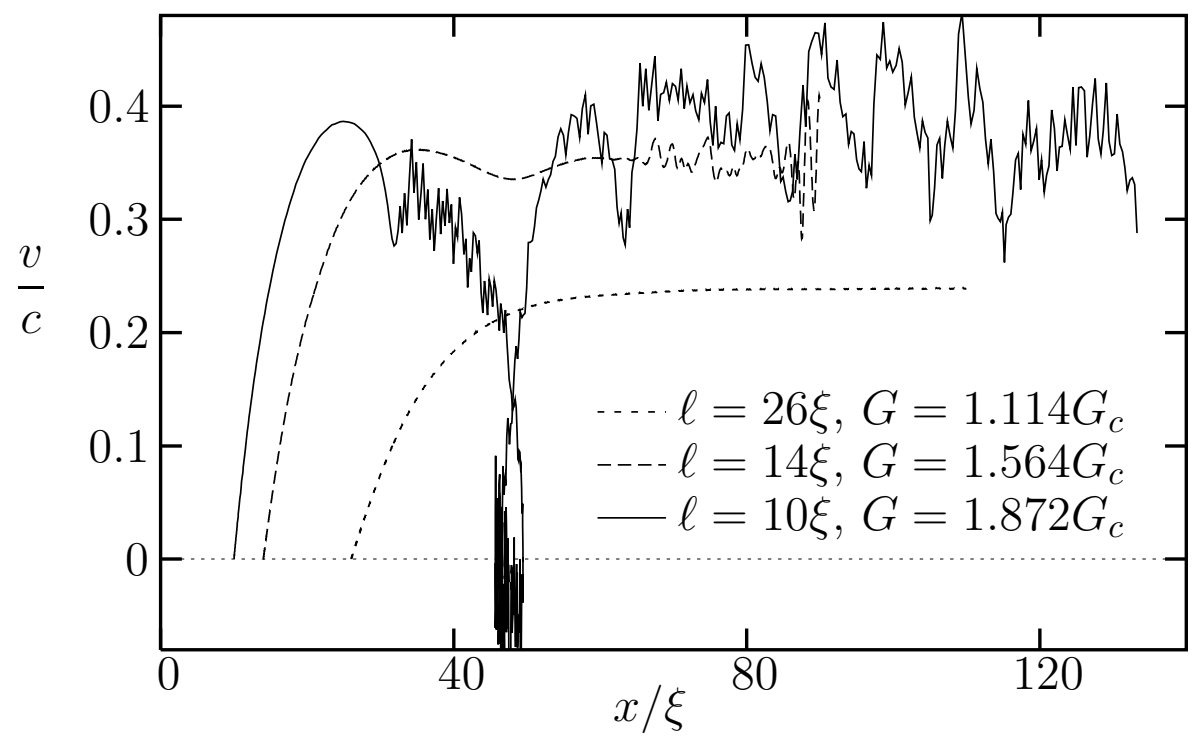

FIG. 1: Crack velocity vs. tip position for cracks accelerating from rest with different initial lengths. $\beta=1, \delta=0$, lattice spacing $d x=0.3 \xi$, and $W=30 \xi$. The velocity for the largest load becomes negative since the symmetric branches retreat to give way to the asymmetric tip-splitting mode with a sinusoidal (snake-like) fracture path.

steady-state or branching to occur such that this feature is not problematic. Furthermore, this radiation is easily suppressed by adding a small Kelvin viscosity to Eq. (2b) with no qualitative change of the results presented here.

We have verified that the important observables converge quickly in the limit of the vanishing lattice spacing, and that we operate in the regime where these observables differ by at most $15 \%$ from their continuum limits for the lattice spacing $d x$ in the range $0.3 \xi$ to $0.4 \xi$.

To study unsteady crack propagation, we use stationary solutions of Eqs. (2) as initial conditions that correspond to cracks of different initial length at rest. For a given load $G$ and corresponding initial crack length $\ell$, these stationary solutions are found by relaxing $u$ with the Gauss-Seidel iteration scheme, which amounts to solving Eq. (2b) without inertia, while $\phi$ is relaxed using Eq. (2a $)$. This procedure yields stationary cracks whose initial length $\ell$ decreases with increasing $G$. We then simulate the full equations of motion with inertia to study accelerating cracks with zero initial velocity. These simulations parallels previous experiments [2] and lattice simulations [4]. To study steady-state features of crack propagation independent of initial conditions, we run long simulations in strips that are 


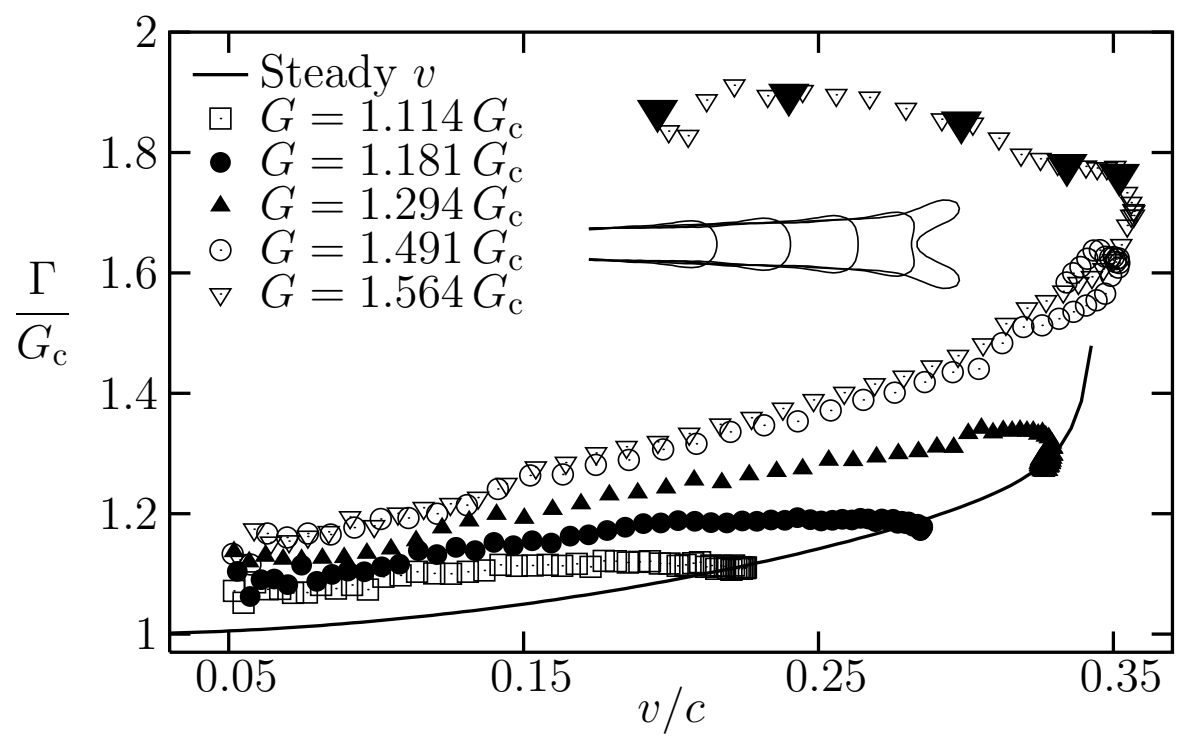

FIG. 2: Fracture energy vs. velocity for the accelerating cracks and the same parameters as in Fig. 1. The solid line corresponds to steady-state crack propagation with $\Gamma=G$ by energy conservation. The five $\phi=1 / 2$ contours shown on the inset correspond to the five large solid triangles. They are equidistant in time separated by $10 \xi / c$. Blunting and subsequent splitting is responsible for the deceleration.

effectively infinite along the propagation direction. To keep the computations tractable, we periodically translate the fields $u$ and $\phi$ by one lattice spacing such that the crack tip remains in the middle of a strip of length much larger than $W$. We have checked that the results of these "tread mill" simulations are independent of boundary effects.

To compute the fracture energy during unsteady motion, we equate $v \Gamma$ with the expression for the energy flow rate to the tip of mode III cracks [3]

$$
v \Gamma=\int_{C} d C\left[\mu \dot{u} \partial_{n} u+v n_{x} \partial_{t}\left(\rho \dot{u}^{2} / 2+\mu|\vec{\epsilon}|^{2} / 2\right)\right],
$$

where $C$ is a closed circuit around the moving tip and $\hat{n}$ is the outward normal to $C$. Since energy is conserved in the model where $\phi=1$, we can equivalently obtain the energy flow rate to the tip by calculating the time rate of change of the total energy energy (elastic plus kinetic) in the region of the system where $\phi$ is larger than a threshold value $\phi_{c}$ arbitrarily close to unity. This quantity is precisely the energy flow rate into the process zone defined as the region where $\phi<\phi_{c}$.

Plots of tip speed versus tip position for cracks accelerating from rest are shown in Fig. [1] for the inertia-dominated regime $\beta=1$. Corresponding plots of $\Gamma$ versus $v$ are shown in 


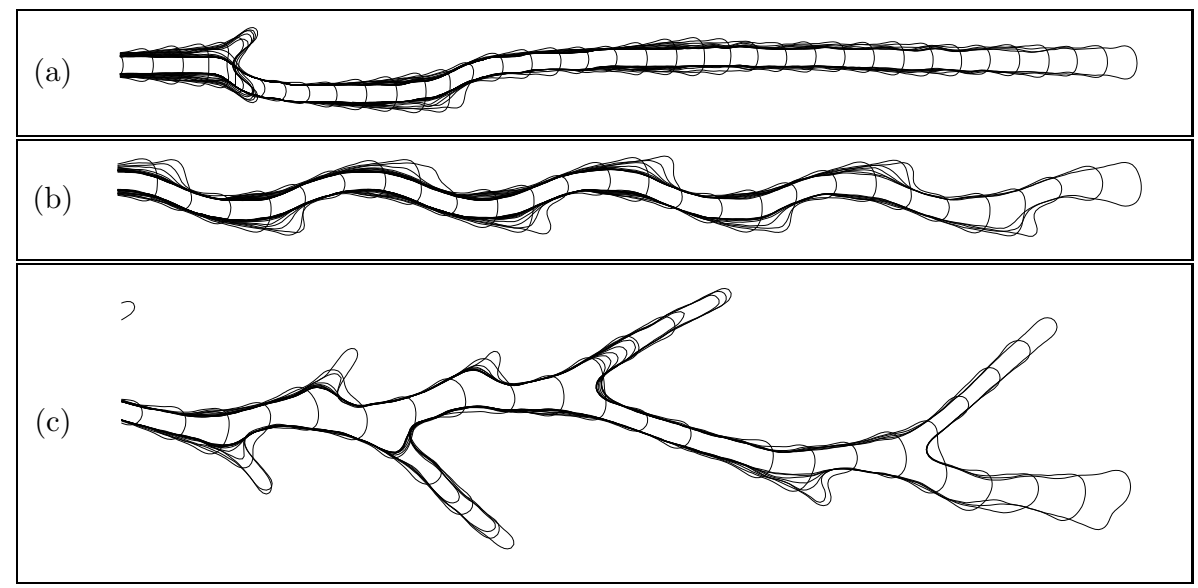

FIG. 3: Contours of $\phi=1 / 2$ separated in time by $\Delta t=10 \xi / c$. All plots are drawn on the same scale for the parameters $\beta=2, \delta=0$, and $W=30 \xi$. (a) Transient branching for $G=1.56 G_{\mathrm{c}}$. (b) Weak periodic branching (the "snake") for $G=1.86 G_{\mathrm{c}}$. (c) Chaotic branching for $G=2.90 G_{\mathrm{c}}$.

Fig. 2. The initial crack acceleration increases with load and the crack tip splits into two symmetric branches above a critical onset load $G_{\text {onset }}$. Cracks for smaller loads which do not split, or split only transiently for $G \sim G_{\text {onset }}$, reach a steady-state propagation velocity that coincides with the steady-state velocity calculated on the tread mill (solid line in Fig. 2). The fact that $\Gamma=G$ in steady-state is a self-consistency check of our method of calculating $\Gamma$ for unsteady cracks.

From the long simulations on the tread-mill, three basic regimes of crack propagation can be distinguished: a stable regime for small loads, where $v$ is constant in time and the crack is rectilinear, an asymmetric tip-splitting ("snake") mode for intermediate loads, where $v$ fluctuates in time around some average value while the crack follows a sinusoidal trajectory, and a chaotic tip-splitting regime for large loads. Examples of these regimes are shown in Fig. 3. The simulations also reveal a strong coupling between the crack tip dynamics and elastic waves that we have not explored in detail. A clear signature of this coupling is the fact that the temporal period of branching in the snake mode is very close to the period $4 W / c$ of the first harmonic standing wave of the strip. The transient coupling of the tip to higher frequency waves is also seen for higher loads in the chaotic branching regime.

The steady state velocity $v$ saturates at some value $v_{c}$. Rectilinear cracks cannot propagate faster than $v_{c}$. We conclude that off-axis branching in the present model is due to the absence of steady-state crack solutions above a critical speed, rather than a linear instability 


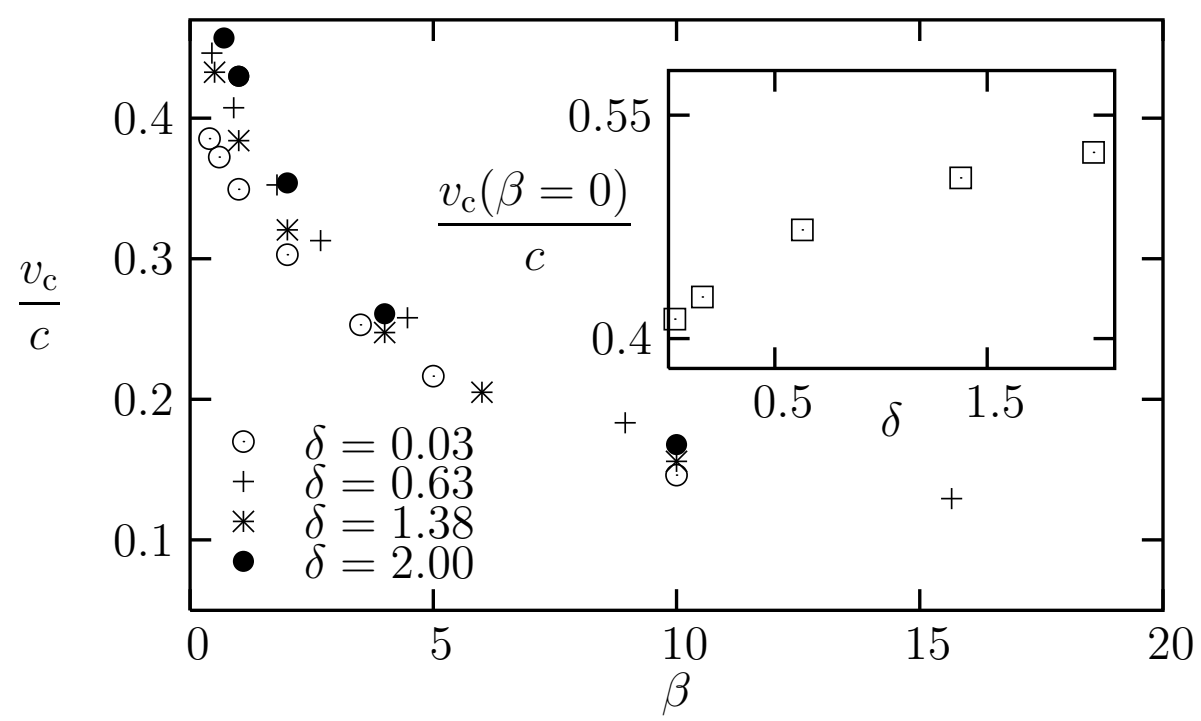

FIG. 4: Limiting speed of crack propagation $v_{c}$ vs. $\beta$ for different values of $\delta$ (or $\widetilde{\gamma}$ given by Eq. 3). Inset: $v_{c}$ in the inertia-dominated $(\beta \rightarrow 0)$ limit vs. $\delta$.

of solutions that exist up to the wave speed, as in the lattice models [10]. The dependence of $v_{c}$ on the parameters of the model is shown in Fig. 4. The maximum crack speed is well defined in the inertia dominated limit $\beta \rightarrow 0$. It grows monotonically with the scaled surface energy $\widetilde{\gamma}($ Eq. (3) $)$ and has a minimum at $\delta=0$ of $v_{c} \approx 0.41 c$. At this speed the linear elastic field in the unbroken material around the crack tip is quasi-isotropic [12]. We therefore conjecture, along the lines of Gao [13], that tip blunting which leads to velocity saturation and ultimately to tip-splitting is due to the relativistic contraction of stress fields in the nonlinear process zone where the sound speed is small due to the softening of the material.

The smallest value of $v_{c} \approx 0.41 c$ in the $\beta \rightarrow 0$ limit is consistent with the calculation of Adda Bedia [14] which shows that tip splitting is energetically possible for mode III fracture for speeds above $0.39 c$ with a maximum angle between symmetric branches of 80 degrees, which is about 10-15 percent larger than the angle in our simulations. It should be noted, however, that this calculation [14] considers a steady-state crack that stops abruptly and splits into two daughter cracks, whereas cracks decelerate before splitting in our simulations. In addition, the onset of branching depends here on the short-scale parameters $\beta$ and $\widetilde{\gamma}$. Therefore, it cannot be predicted from purely energetic considerations.

To interpret our results for accelerating cracks, it is useful to first derive an analytic 
expression for the speed of steady-state cracks close to the Griffith threshold. Energy balance for steady-state propagation implies that the stored elastic energy ahead of the crack in excess of twice the surface energy must be dissipated in the process zone. This implies that

$$
v\left(G-G_{c}\right)=-\frac{d E}{d t}=\chi^{-1} \int d \vec{x}\left(\frac{\partial \phi}{\partial t}\right)^{2},
$$

where the second equality follows from Eqs. (2) with no energy flux through the boundaries. In a coordinate system $\left(x^{\prime}=x-v t, y^{\prime}=y\right)$ translating with the tip, $\partial \phi / \partial t=-v \partial \phi / \partial x^{\prime}$. Thus the steady-state velocity of rectilinear crack propagation is

$$
\frac{v}{c} \approx \frac{2 \tilde{\gamma}\left(G / G_{c}-1\right)}{\beta I}
$$

where $I \equiv \int d x^{\prime} d y^{\prime}\left(\partial \phi / \partial x^{\prime}\right)^{2}$ is a dimensionless integral factor of order unity that depends on the profile of $\phi$ for a stationary crack at $G \approx G_{c}$. This prediction is consistent with the simulation results that show a steeper increase of velocity with load for smaller $\beta$.

The fact that the $\Gamma-v$ plots for different accelerating cracks in Fig. 2 do not superimpose on the steady-state curve clearly shows that the speed of these cracks is not uniquely determined by the energy flow rate to the tip. In our model, accelerating cracks require more energy per unit length of advance than steadily propagating cracks. This extra energy is reversibly stored in the process zone to be either consumed by branching or radiated away later. This effect is exacerbated for large loads and causes the crack to decelerate before branching. This deceleration is marked by the overshoot of the velocity plotted as a function of tip position (Fig. 1) and the fact that the corresponding $\Gamma-v$ plot becomes double-valued (Fig. 2). A qualitatively similar overshoot of the velocity was observed experimentally for very large accelerations in brittle fracture of glass [2], albeit for much larger systems than in the present simulations.

To estimate when crack acceleration should be important in our model, we compute the change of the velocity over a distance comparable to the process zone. Crack's acceleration should be irrelevant when this change is small compared to the wave speed, or $\xi d(v / c) / d x \ll$ 1. For cracks accelerating from rest, let us assume that Eq. (6) gives the instantaneous speed of the accelerating crack if $G$ is taken to mean the instantaneous elastic energy release rate $\Gamma$ which varies as $d \Gamma / d x \sim\left(G-G_{c}\right) / W$ as the crack extends into the stressed strip. This implies that the acceleration is small when

$$
\frac{W}{\xi} \gg \frac{G-G_{c}}{G_{c} \beta} \text {. }
$$


We find that acceleration remains important in our simulations even for $W / \xi=100$ when $\beta$ is of order unity. Since in experiments $G$ can be up to a hundred times larger than $G_{c}$ and $\beta=c \tau / \xi$ can be much smaller than unity, acceleration may be important even in macroscopic strips several orders of magnitude larger than the process zone size. We conclude that the validity of the continuum theory of brittle fracture depends not only on the ratio of the system size to the process zone size, but also on the importance of inertia relative to bond breaking dissipation measured here by $\beta$.

This conclusion does not conflict with the fact that this theory was recently validated by lattice simulations in the limit of zero dissipation [4]. In these simulations, the velocity jumps discontinuously to a fraction of the wave speed and the slope of the velocity-load relation is finite for velocities larger than this fraction. Therefore, $1 / \beta$ should be replaced by $d(v / c) / d\left(G / G_{c}\right) \sim 1$ in the above estimate leading to Eq. (77). In contrast, for the velocity range from zero to a fraction of the wave speed pertinent to experiments, the continuum theory will break down for accelerating cracks in the $\beta \rightarrow 0$ limit of the present model for any finite system size. The crack dynamics is independent of $\beta$ and well-defined in this limit.

Clearly, the incorporation of more realistic microscopic mechanisms of failure in the present phase-field approach and the extension to mode I remain needed to make contact quantitatively with experiments.

This research is supported by U.S. DOE Grant No. DE-FG02-92ER45471.

[1] L. B. Freund Dynamic fracture mechanics (Cambridge University Press, 1990).

[2] E. Sharon and J. Fineberg, Nature 397, 333 (1999).

[3] J. D. Eshelby, J. Mech. Phys. Solids 17, 177 (1969).

[4] D. A. Kessler and H. Levine, Phys. Rev. E 68, 036118 (2003).

[5] T. Cramer et al., Z. Metallkd. 90, 686 (1999); Phys. Rev. Lett. 85, 788-791 (2000).

[6] K. Ravi-Chandar and W. G. Knauss, Int. J. Fracture 25, 247 (1984).

[7] F. F. Abraham et al., Phys. Rev. Lett. 73, 272 (1994); D. Holland and M. Marder, Phys. Rev. Lett. 80, 746 (1997).

[8] J. S. Langer, and A. E. Lobkovsky, J. Mech. Phys. Sol. 46, 1521 (1998).

[9] M. Marder and X. Liu, Phys. Rev. Lett. 712417 (1993). 
[10] D. A. Kessler and H. Levine Phys. Rev. E 63, 016118 (2001).

[11] A. Karma et al., Phys. Rev. Lett. 87, 045501 (2001).

[12] E. Y. Yoffé, Philos. Mag. 42, 739 (1951).

[13] H.-J. Gao, J. Mech. Phys. Solids 44, 1453 (1996).

[14] M. Adda-Bedia, J. Mech. Phys. Solids, in press (2003). 